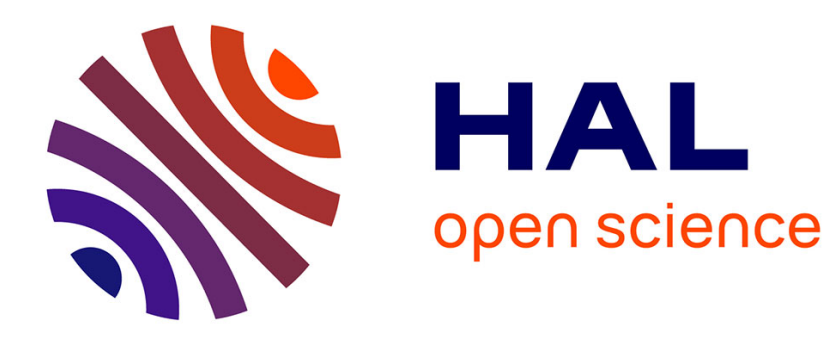

\title{
L'abstinence des céréales chez les taoïstes
}

Jean Levi

\section{To cite this version:}

Jean Levi. L'abstinence des céréales chez les taoïstes. Études Chinoises, 1982, I, pp.3-47. halshs00178504

\section{HAL Id: halshs-00178504 \\ https://shs.hal.science/halshs-00178504}

Submitted on 11 Oct 2007

HAL is a multi-disciplinary open access archive for the deposit and dissemination of scientific research documents, whether they are published or not. The documents may come from teaching and research institutions in France or abroad, or from public or private research centers.
L'archive ouverte pluridisciplinaire HAL, est destinée au dépôt et à la diffusion de documents scientifiques de niveau recherche, publiés ou non, émanant des établissements d'enseignement et de recherche français ou étrangers, des laboratoires publics ou privés. 


\section{L'ABSTINENCE DES CÉRÉALES CHEZ LES TAOÏSTES}

Tout mouvement se définissant à la fois en tant que secte religieuse, milieu philosophique et groupe politique cherche à se particulariser en soulignant ses différences avec le corps social qui lui a donné naissance et en s'opposant aux groupes dont la société se compose. Et il le fait par un ensemble de comportements, inséparables de différents codes, qui, pour reprendre la formulation de M. Détienne à propos du pythagorisme, «imposent au groupe son faciès et sa configuration propres ${ }^{1}$.

Parmi cet ensemble d'attitudes, de manière d'être et d'agir, les conduites alimentaires occupent une place centrale. D'une part, parce qu'elles constituent une activité générale et quotidienne, d'autre part, parce qu'elles sont sous-tendues par un réseau de relations et d'oppositions reflétant l'armature du système philosophico-religieux dans lequel elles s'inscrivent et qu'elles prolongent. Les manières de manger sont à la fois matière à penser et manière de penser. En elles se vérifie quotidiennement une façon d'appréhender le monde.

Or l'un des traits les plus surprenants du taoïsme, qui, à l'instar de mouvements philosophico-religieux tels le bouddhisme, le

1. Cf. M. Détienne (1970, p. 141). 
pythagorisme ou l'orphisme, distingue des aliments purs, bons à manger, et des aliments impurs à l'intérieur des nourritures, est qu'il proscrit les céréales, une nourriture dont il semblerait, à première vue, qu'on ne puisse se passer, dans une société essentiellement agricole, alors qu'il prétend obtenir par ce moyen l'immortalité matérielle.

Quelle est la signification de telles conduites? Comment expliquer que des pratiques qui visaient à obtenir un résultat concret aient pu se perpétuer si longtemps quand elles aboutissaient le plus souvent à des résultats opposés à ceux promis? Un tel type de comportements ne reposait-il pas moins sur un souci d'efficacité que sur un jeu complexe d'oppositions et de relations symboliques? En d'autres termes, les représentations mentales dans lesquelles elles s'inscrivaient n'en garantissaientelles pas l'efficacité quel que pût être le résultat réel de leur confrontation avec les faits?

Cette étude se propose d'apporter des éléments de réponses à ces interrogations en dégageant l'armature symbolique commune au taoïsme religieux et au taoisme ancien et en montrant que pratiques diététiques et conceptions métaphysiques ne sont que deux manifestations complémentaires d'une même structure. En effet, les conduites alimentaires des taoïstes ne peuvent s'interpréter que dans un système global, et poser la question des nourritures dans le taoïsme, ce n'est pas traiter d'un aspect insolite ou accessoire mais se situer au cœur même de sa problématique ${ }^{2}$.

2. A notre connaissance, peu d'études ont été consacrées au problème de la diététique taoïste. H. Maspéro (1971, pp. 481-589; 365-380), mentionne le régime alimentaire taoïste en passant, dans des analyses essentiellement descriptives. M. K. Schipper (1982, pp. 216-221), dans son bel ouvrage sur le taoïsme, reprend la question dans une perspective ritualiste. Et surtout $R$. Stein dans ses cours dispensés au Collège de France en 1971- 
1. Les céréales: Les ciseaux qui coupent la vie

Vers les III $^{\mathrm{e}}-\mathrm{IV}^{\mathrm{e}}$ siècles de notre ère, on voit fleurir toutes sortes de pratiques étranges dont certaines visaient à obtenir l'immortalité matérielle. Par des techniques gymniques, respiratoires, alchimiques, diététiques, etc., l'adepte remplaçait son corps mortel par un embryon d'immortalité. Nombreuses et complexes, ces pratiques ont beaucoup varié selon les écoles et les époques. Parmi elles les pratiques diététiques ont joué un rôle si important que c'est à son régime alimentaire que l'on reconnaît le taoïste.

Dès une date ancienne l'abstinence des céréales dénonce d'emblée le disciple de Lao zi. Wang Chong, rationaliste du $1^{\text {er }}$ siècle de notre ère, combat la prétention de certains d'entre eux à accéder à l'immortalité en interrompant la nourriture céréalière : «Ceux qui refusant les céréales ne mangent rien sont versés dans le taoïsme... Ils prétendent que les ascètes tels Wang $\mathrm{Zijiao}$ qui les proscrivaient de leur alimentation et se nourrissaient autrement que le reste des mortels avaient une longévité tout autre et qu'après avoir traversé les siècles ils devenaient immortels (Lunheng, p. 113). Le Lüshi chunqiu de Lü Buwei fait allusion à un sage qui «pratiquait l'art de ne pas manger les céréales» $(14.23 \mathrm{~b})^{3}$.

1972 et 1972-1973 sur le système des nourritures en Chine s'est livré à une analyse approfondie de la signification symbolique des comportements alimentaires des taoïstes. Le présent article développe et systématise un certain nombre de ses idées.

3. Le Shi $j i$ (2048; Cf. aussi Hanshu : 2037) affirme que Zhang Liang après avoir contribué à installer les Han sur le trône aurait abandonné le commerce des hommes pour s'initier à l'art d'interrompre les céréales et pratiquer la gymnastique daoyin tableau des différentes postures de la gymnastique daoyin datant du III siècle av. J.C., mis à jour par les fouilles de Mawangdui, s'ouvre sur un paragraphe consacré à l'interruption des céréales et à l'ingestion du souffle (Wenwu 1975, p. 1). Zhuang zi fait allusion au même anachorète que le Lüshi chunqiu en se contentant d'affirmer qu'il se sustentait de baies sauvages (Pères, p. 361). Dans le Zhuangzi, toujours, est évoquée une île paradisiaque dont les habitants «ne mangent pas les cinq céréales et aspirent le vent et la rosée 》 (Pères, p. 211). 
Pratique attestée par des textes d'une époque relativement reculée, l'abstinence des céréales apparaît dans les contes et les biographies taoïstes comme l'un des moyens d'échapper au vieillissement. Tantôt, comme dans le Bowuzhi 博物志 (2.p.15), c'est un homme qui, tombé dans un abîme, apprend des serpents et des tortues l'art de tendre le cou matin et soir vers l'orient. Il n'a plus faim ni soif, son corps devient lumineux et léger; il peut, après plusieurs années, s'élever du gouffre et retourner chez lui. Mais à peine recommence-t-il à ingérer des céréales qu'il retrouve son apparence ordinaire. Tantôt, comme dans un récit du Baopuzi (11, p. 208), c'est une concubine obligée de se réfugier dans les montagnes pour échapper aux troubles survenus sous le règne du prince de Qin qui est initiée à la diététique taoïste par un immortel alors qu'elle était sur le point de mourir d'inanition; deux cents ans plus tard, elle est capturée par les chasseurs et se remet à manger des céréales. Très rapidement, elle devient une petite vieille décrépite et meurt ${ }^{4}$.

Remontant aux sources mêmes du taoïsme, l'abstinence des nourritures céréalières a persisté tout au long de son histoire. Elle ne fut jamais exclue par aucun courant, même les

4. On pourrait multiplier les exemples. Le Soushenji (1.1a) cite le cas de Chijiang qui « ne mangeait pas les cinq céréales et était capable de monter et de descendre au gré du vent et de la pluie ". Le Shenxian zhuan contient de nombreuses références à des taoïstes auxquels l'abstinence des céréales procure une longévité exceptionnelle : Chen Jian interrompt les céréales et devient si léger qu'il semble voler en marchant (6.4b); Wang Zhen s'intéresse au Dao à l'âge de 79 ans, pratique la respiration embryonnaire, supprime les céréales pendant trente ans. Il obtient un visage d'enfant et un teint de lys, puis devient immortel après s'être retiré sur la montagne Nüji. Cf. aussi ibid. $(10.3 b ; 9.1 b ; 10.4 b)$. Parfois, c'est à leur capacité à se passer de grains pendant de très longues périodes qu'on reconnaît les taoïstes (ibid. 5.3a; 7.3b). 
pius influencées par le bouddhisme ${ }^{5}$. Lorsque le taoïsme s'organise en communautés laïques, à la fin des Han et sous les Six Dynasties, le refus d'ingérer des céréales, s'il ne se traduit plus par aucune pratique effective n'en demeurera pas moins dans les hagiographies des saints patrons, réels ou mythiques, des différents courants ce qui marque de façon définitive l'entrée en taoïsme ${ }^{6}$.

Cette interdiction est absolue. Il ne saurait y avoir de vie éternelle pour le mangeur des nourritures céréalières. Le Daoyou jing 大 有 终 est formel : 《Les céréales sont les ciseaux qui coupent la vie. Elles pourrissent les cinq viscères... Si un grain entre dans ta bouche n'espère pas la vie éternelle !» (Taisho 52 no 2103, 148B). Et dès le $\mathrm{II}^{\mathrm{e}}$ siècle av. J.-C. le Huainanzi cite un dicton devenu par la suite un lieu commun de la classification des etres et des nourritures : "Ceux qui mangent des céréales sont intelligents mais meurent tôt; ceux qui ne mangent rien sont des immortels » $(4, \text { p. } 131)^{7}$.

Il nous faut tout de suite ajouter que certains textes ne font pas de l'interruption des céréales une condition suffisante pour devenir immortel. Pour le Baopuzi elle ne ferait pas partie des pratiques d'immortalité, et seuls les faux taoïstes s'y livreraient : «Il est évident que les diverses sortes de taoïstes qui existent de nos jours n'obtiendront jamais l'immortalité à moins qu'ils ne connaissent

5. Un cas remarquable est celui du Shengxuan jing 茾玄经 du courant Lingbao 霆䨳 qui reprend et intègre toute la phraséologie bouddhique mais mentionne néanmoins, parmi les enseignements de base, l'abstinence de céréales (cité in Dadeo tong xuan yao ch. 14.1.65 fd. Zhen Songtang 真松堂 etShengxuan jing:4a).

6. Dans l'immense recueil en 53 juan de Zhao Daoyi réunissant toutes les biographies de saints taoïstes des origines au XIII ${ }^{\mathrm{e}}$ siècle, le Lishi zhenxian tidao tongian, pour presque tout immortel, quelle que soit l'école à laquelle il appartient et son époque l'abstinence des céréales est mentionnée.

7. Cf. aussi Bowuzhi (7, p. 40); Yansheng yannian lu citant le Shennong ji 神 表記 (1.1a). 
la Grande Recette pour fondre le cinabre. Seraient-ils capables de faire se lever les morts par leurs drogues, de s'abstenir de céréales pendant plusieurs années sans souffrir de la faim, de mettre les esprits à leur service... qu'ils n'accroîtront en rien leur longévité » (neibian, 15, p. 263). Ailleurs, il dit en réponse à une question sur le refus d'ingérer les nourritures céréalières que celui-ci ne peut rien contre le vieillissement (neibian, 15, pp. 273-274).

Le Shenxian zhuan, sans être aussi catégorique, insiste à plusieurs reprises sur le fait que l'interruption des céréales ne suffit pas à devenir immortel pas plus que l'avalement du souffle ( $3.2 \mathrm{~b}$; $3.4 \mathrm{a} ; 4.3 \mathrm{~b}$ ). Un certain nombre de textes ne mentionnent d'ailleurs pas l'abstinence des céréales parmi les règles d'hygiène ou de diététique permettant d'obtenir l'immortalité ou tout au moins de prolonger la vie ${ }^{8}$. L'abstinence ne serait donc le fait que de certains groupes et non un trait constitutif du taoïsme. En réalité, même contestée par certains, cette pratique n'en demeure pas moins l'un des principes généraux de l'ascèse taoïste. Et ceux-là mêmes qui lui dénient toute vertu se voient obligés, comme par devers eux, de lui laisser une place de choix dans leurs écrits. C'est ainsi qu'un grand nombre de passages du même Baopuzi consacrés aux drogues d'immortalité font référence à l'abstinence des céréales (11, pp. 200, $202,203,206,208)^{9}$. On trouve encore de nombreuses références aux Trois Cadavres (San shi $\equiv \mathcal{P}$ et aux moyens de les

8. Le Zhenzhong $i i$ (p. 3a) parle de supprimer les pâtés de poisson et tout ce qui a une odeur de viande crue. Pour le Pengzu sheyang yangxing lun (p. 2b), la diététique taoïste consiste à régler ses désirs; à ne pas abuser d'une nourriture trop riche, à accorder ce que l'on mange avec les saisons et associer judicieusement les mets entre eux.

9. Au chapitre 15 (pp. 267-274) bien que les citant comme « les racontars d'une école ", il se croit obligé de retracer en détails toutes les pratiques qu'entraîne le refus d'ingérer les grains. Au chapitre 20 (p. 395), il raille un faux taoïste qui pratiquait l'abstinence des céréales sans connaître les drogues qu'il fallait leur substituer. 
évacuer ${ }^{10}$. Or on verra que ceux-ci sont étroitement liés aux céréales. De même, le Zhenzhongji, après avoir donné des principes de modération dans l'ingestion des nourritures, présente une série de recettes permettant de "détruire les vers des céréales 》 (shamie guchong 殺减款蟲) et ajoute que tant qu'on n'a pas expulsé les Trois Vers ni interrompu la manducation des grains, les drogues ne servent à rien $(24 a, 24 b)$.

En réalité, il apparaît que l'abstinence des céréales est la pratique ancienne et qu'elle a été peu à peu, sinon totalement abandonnée, tout au moins reléguée au second plan au profit des pratiques alchimiques ${ }^{11}$.

La défense de manger des céréales n'est pas le seul interdit alimentaire mentionné par les textes taoïstes. Certaines diètes proscrivent la viande, les saveurs fortes, l'alcool ${ }^{12}$. Mais celles-ci n'ont rien de comparable avec le refus de manger des céréales.

10. Au chapitre 11( p. 200) il est question d'une drogue qui évacue les Trois Cadavres et fait descendre des « cuisines de voyage " (Cf. aussi 15, p. 279, 11, pp. 203215).

11. La propagation du bouddhisme qui interdit le meurtre des êtres vivants et la manducation des viandes a dû jouer un rôle. On notera cependant que le refus de certaines nourritures dans le taoïsme n'est jamais dicté par des raisons morales mais toujours hygiéniques. Il faut aussi tenir compte de la difficulté qu'il y avait à concilier l'interdiction théorique des céréales et sa réalisation pratique. C'est ainsi que celles-ci peuvent entrer dans la composition de certaines drogues censées soit se substituer à elles, soit éliminer les Trois Vers qu'elles nourrissent à l'intérieur de l'organisme ! Un autre moyen de concilier la pratique avec la théorie a été de s'abstenir durant certaines périodes. Des traités donnent le nom des médecines permettant de revenir à l'alimentation normale sans troubles pour l'organisme (Cf. Taiqingiing duangu fa, 11b).

12. Cf. entre autres H. Maspéro ( 1971 , p. 368) et Zhenzhong $j i$ (7a). On pourrait aussi citer le Laozi. Mais le texte n'est pas clair. Il faut sans doute comprendre, par cinq saveurs 五味 que l'on ne doit pas se laisser détourner par les mets trop riches de la méditation intérieure. Il s'agit donc plutôt d'une critique du sensualisme que des mets épicés (Pères, p. 27). 
Beaucoup plus floues, moins impératives, elles ne sont pas la marque, la caractéristique de l'adepte. De nombreux taoïstes ont usé, sinon abusé de l'alcool. Mieux, l'ivresse est recommandée car elle procure un état voisin de l'extase ${ }^{13}$. Quant à la viande, son statut est des plus ambigus. Si quelques textes parlent des effets nocifs des viandes riches et grasses ${ }^{14}$, la viande séchée est l'une des nourritures de l'anachorète taoïste, et la santé de l'adepte se manifeste dans ses appétits carnivores ${ }^{15}$.

Mais ce qui fait que l'abstinence des céréales constitue la pierre angulaire de tout le système diététique des taoïstes, c'est que ceux-

13. Le Liezi (Pères, p. 87) assure qu'un homme tombant de son char en état d'ébriété ne se fera aucun mal car sa puissance vitale (shen 神) est totale. Même interdit, le vin ne fait pas l'objet d'une rếglementation aussi stricte que les céréales. Le Shenxian zhuan (6.2a) parle d'un taoïste, Kong Yuan fang, qui ne buvait jamais plus d'un sheng 牛 de vin par repas. Ailleurs un taoïste ne peut devenir un immortel céleste en raison de son amour pour la dive bouteille. Il n'en devient pas moins un immortel terrestre. A cinq cents ans il avait un visage d'adolescent. Le seul inconvénient causé par son vice était ses cheveux blancs (10.2b). Dans d'autres biographies du même recueil, la vertu de certaines drogues est soulignée par la tolérance à l'alcool qu'elles procurent (10.2b).

14. Que pour certains taoïstes la viande ne soit nullement interdite apparaît a contrario dans un passage du Baopuzi $(15$, p. 273) où sont pris à parti ces prétendus taoïstes qui pensent que seule l'abstinence des céréales suffit. Ge Hong dit qu'ils ne diffèrent en rien de ces goinfres qui s'empiffrent de viande ou des piliers de cabarets. L'idée de la nocivité d'une alimentation trop riche en viandes est ancienne. Elle apparaît dans le Hon Fei zi: 8, p. 121 ; fu xiangmei cuiwei, houjiu feirou gankou bingxing 夫香美脆味厚海 肥肉甘病形 où se sent une assez forte résonance taoiiste. Ou encore dans le Qifa (Wenxüan 34, p.748) : gancui feinong, mingyue : «fu chang zhi yao》 甘脆肥 滕命日：腐腸之菜。

15. La viande séchée est une nourriture de choix chez les taoïstes. Elle figure dans les repas organisés par les divinités et les adeptes (Shenxian zhuan, 3.2a; 2.2b). Dans le même ouvrage est mentionné un taoïste, $\mathrm{Li}$ Yiqi, qui habite dans une caverne, porte, hiver comme été, un vêtement non doublé et ne se nourrit que de jujubes, de châtaignes et de viande séchée ( $b u$ 脯) (3.3b). Ailleurs, encore, un adepte monte dans les paradis taoïstes et reçoit au moment de prendre congé d'énormes jujubes et des tranches de viande séchée $(8.5$ b). Zhao Ju pouvait croquer les os des lièvres et des faisans à l'âge de 70 ans. Il vécut parmi les hommes jusqu'à l'âge de trois cents ans avant de disparaitre dans les forêts $(3.4 \mathrm{a})$. 
ci ont élaboré autour des grains une véritable mythologie démoniaque.

Non seulement les chercheurs d'immortalité refusent de les ingérer, mais ils éprouvent à leur égard une véritable horreur. Non contents de proscrire absolument et formellement l'ingestion de grains, les manuels de longue vie expliquent en détail les raisons de cette prohibition. Les céréales sont interdites parce qu'elles sont germe de mort; elles affaiblissent l'organisme et provoquent toutes sortes de troubles. Ne suffit-il pas pour s'en convaincre d'observer l'effet qu'elles produisent sur ces êtres sains et libres qui n'en consomment ordinairement pas : "Quand les barbares mangent du riz, ils deviennent lépreux; les chevaux ont le pied alourdi quand ils mangent du grain et les oies sauvages ont des tumeurs sur tout le corps quand par malheur elles ingèrent ce poison ${ }^{16}$. En même temps qu'elles corrodent l'organisme et font fuir les esprits vitaux par leurs exhalaisons putrides ${ }^{17}$, les cérérales engraissent à l'intérieur du corps les Trois Vers (San chong三虫) ou Trois Cadavres (San shi 三 $\mathcal{P}$ ), principes de pourriture, de corruption ou de mort ${ }^{18}$. En effet les Trois Vers ou Trois Cadavres, gorgés des effluves pestilentiels des céréales sont eux-mêmes une véritable pourriture. On les retrouve dans les matières fécales, associées étroitement à la mort ${ }^{19}$. Mais il y a mieux : les Trois Vers, génies

16. Xi Kang : Da nanyangsheng lun (Luxun quanji IX).

17. H. Maspero (p. 368) citant le Livre de la cour jaune: «leur puanteur trouble les esprits et le souffle embryonnaire cesse ».

18. Le thème des Trois Vers est ancien. Le Lunheng (16, pp. 253-254) fait à la fois allusion aux Trois Vers : «Dans le ventre de l'homme il y a trois vers... qui rongent les intestins» et aux vers qui naissent des céréales, surtout par temps humide. Ceux-ci sont semblables aux $k u$, vermine de pourriture.

19. Le Huangting jing (nei) (Concordance : 3.3) dit que celui qui ne mange pas de céréales est libre de pourriture. Le commentaire de Liang Qiuzi explique qu'en supprimant les céréales on se libère des immondices que le corps renferme. Ge Hong dans le 
retenus prisonniers à l'intérieur du corps de chaque homme, cherchent à hâter la fin de l'organisme qui les tient enfermés pour conquérir leur liberté et devenir des esprits que l'on honore par des sacrifices et des offrandes. Aussi, épiant les fautes des hommes, ils en dressent l'inventaire et, le $57^{\mathrm{e}}$ jour de chaque cycle de 60 jours, ils montent au ciel faire un rapport au Directeur du Destin qui retranche un certain nombre d'années de vie aux humains ${ }^{20}$.

En refusant les céréales, c'est en fait une certaine forme de cuisson que les sectateurs de Lao zi condamnent. En effet les céréales sont assimilées à une cuisson sur le double plan des représentations mythiques et du champ sémantique. Le divin laboureur Shennong, souverain qui apporta aux hommes la culture du grain entretient des rapports privilégiés avec l'élément igné : surnommé Yandi 炎帝 l'Empereur Ardent, ou Chidi 赤帝 l'Empereur rouge, il administre le midi, règne sur le feu et préside au sacrifice au fourneau en tant qu'esprit du foyer (Huainanzi 13, p. 512). Dans son activité agricole il est associé au feu, et ce, de deux façons ; d'une part l'invention des céréales est calquée sur celle du foret à feu : à l'instar de cette dernière elle aboutit à la transformation du $\mathrm{cru}^{21}$, d'autre part, sur le plan technique, l'activité agricole est

Baopuzi $(15$, p. 267) cite les conceptions de certaines sectes pour lesquelles «ceux qui veulent obtenir la longue vie doivent avoir les intestins purs». Le Wufu $x u(2.23 b-24 a)$ affirme que les Trois Vers, nourris par le souffle des céréales, sortent pourris (lan chu 䍩出).

20. Cf. H. Maspero, pp. 367, 336-366. Cf. aussi Baopuzi (11, p. 215). Le Shenxian zhuan (3.2b) ajoute que les mauvais rêves proviennent des combats que se livrent les Trois Cadavres et les esprits vitaux à l'intérieur du corps. Certains textes font allusion à des procédés qui permettent d'empêcher les Trois Vers de sortir faire leur rapport au Directeur du Destin et donc d'obtenir une longévité exceptionnelle (Cf. Sandong shuji zashuo citant le Chisu yujue 赤素王訣 4a.

21. "Avant le peuple se nourrissait de viande crue et buvait le sang des animaux. Alors Shennong survint,... Il apprit aux hommes à cultiver le grain " (Huainanzi, 19, p. 735). Cf. aussi Hanfeizi, 49, 1040, Fengsu tongyi, 1, p. 3. 
tributaire de la maitrise du feu : anciennement on pratiquait l'agriculture sur brûlis. La chasse d'hiver (shou 狩) accompagnait l'incendie de défrichement. Les connexions entre les trois activités : défrichement par le feu, chasse et agriculture apparaissent de façon particulièrement nette dans le mythe d'aménagement des eaux par $\mathrm{Yu}$ le Grand ${ }^{22}$. Il y a donc identité fonctionnelle. Cuisson des viandes et culture des cérérales mettent fin au manger-cru en même temps que toute culture n'est possible que si elle est précédée d'un feu. Cette corrélation entre les céréales et le feu se retrouve dans d'autres configurations sémantiques. Passant en revue les différentes tribus qui peuplent les quatre orients, le Liji parle de ces barbares qui mangent la nourriture sans la cuire ou ne mangent pas le grain : "A l'Est on les appelle les Ti... certains avalent les aliments sans les cuire. Au Sud on les appelle les Man... certains avalent les aliments sans les cuire. A l'Ouest on les appelle les Rong... certains mangent sans céréales. Au nord il y a les Ji... certains mangent sans céréales ${ }^{23}$. Les quatre phrases sont symétriques. Buhuo 不 $火$ 《sans cuisson» a la même valeur adverbiale que buli 不粒 《sans céréales». Les deux expressions sont équivalentes. Elles traduisent deux modes du manger-cru. L'un est la méconnaissance de la cuisson des céréales au double feu du soleil et du fourneau; l'autre de la cuisson des viandes à la flamme.

22. Le mythe est reproduit dans de nombreux classiques : Cf. Shiji : 83, pp. 154155 (Mémoires : I, p. 209, Annales : 5, p. 50). Sur l'analyse du mythe, cf. H. Maspero, 1971, pp. 227, 231. La coutume de sortir le feu au printemps et de le rentrer en hiver, le parallélisme établi entre les quatre saisons et les différentes sortes de bois qu'on utilise à chacune d'elles pour tirer le feu nouveau sont autant de vestiges de cette antique collaboration (Cf. Lushi, qienji, 5.4b; Tchéou li, XXX, 194).

23. Liji (Mémoires: 3, p. 295). Dans le Dadai liji il n'est question que des peuples qui mangent sans céréales. Ce qui montre bien que les deux expressions sont interchangeables. 
Or le feu lui-même appelle des visions de décrépitude et de mort. C'est du moins ce que suggère un passage du Lunheng (7, p. 107) dans lequel Wang Chong compare la verdeur de la jeunesse à de la viande ou du poisson cru, tandis que l'âge mûr évoque les viandes cuites ou grillées par l'action d'une flamme et qu'il est impossible de ramener à leur état primitif. La sénescence, telle une cuisson, macère les chairs et les corrompt. De même, le feu de cuisine hâte la fin en rognant des années de vie. Tout comme les Trois Vers, le Dieu du fourneau (zaoshen 灶神) qui est aussi Siming 司 命 Directeur du Destin, rend compte des fautes de la famille détentrice, et celle-ci se voit retrancher des années de vie en proportion de ses fautes (Stein 1970, p. 1282). Certaines prières quand on va aux latrines laissent soupçonner une assimilation des latrines au fourneau. Dans un court texte intitulé : «Procédés pour augmenter le nombre d'années qui vous ont été allouées » Dongxuan lingbao zhenren xiuxing yangnian yicou fa au paragraphe «prières aux latrines", figure une formule qui donne clairement à entendre que la déesse qui préside aux latrines est Siming, Directeur du Destin, et peut accorder ou retrancher des années de vie (11b). Or le Directeur du Destin est aussi Dieu du foyer. Les affinités entre Dieu du foyer et les immondices se marquent encore dans les liens qu'il entretient avec le Dieu des richesses (ou de la pauvreté) étroitement associé aux détritus, aux latrines et aux balayures (Stein, 1970, p. 1296).

Ainsi donc, activités culinaires, feu de cuisine ou céréales, entraînent l'homme dans l'univers de la putréfaction et de la mort. Toute cuisson cache en elle un principe de corruption. Le mot lan 爛 《bouilli, cuit», signifie aussi tout ce qui est trop cuit, blet, pourri, ou est principe de putréfaction ${ }^{24}$. Et si le Dayou jing

24. Le Wufu $x u(2,23 b-24 a)$ emploie l'expression lan pour décrire l'aspect sous lequel sont évacués les Trois Cadavres. 
nous dit que les céréales «pourrissent» $f u$ 腐 les intestins et les viscères (cité in Xiaodao lun. Taisho, 52, no 2103, p. 146B), Xi Kang emploie concurremment à des expressions connotant la putrescence des expressions qui renvoient au vocabulaire culinaire pour décrire l'effet pernicieux des mets trop riches : « les viandes grasses cuisent (qian 前 les entrailles, les vins capiteux font bouillir (zhu 煮, l'estomac et les intestins (Yangsheng lun, Luxun Quanji, p.55). La digestion, considérée aussi comme une cuisson, est également cause de mort : le Baopuzi (15. p. 269) donne certaines recettes qui permettent d'ingérer la nourriture sans l'assimiler ${ }^{25}$. La respiration embryonnaire (taixi 胎息), la circulation du souffle sont bénéfiques parce qu'elles constituent des moyens de s'alimenter sans digérer.

\section{II - Nourritures sauvages - nourritures célestes}

En《coupant les céréales》(duangu断教 ou juegu纯乹 ou encore quegu 仰勃 pour échapper à la mort et en choisissant d'autres nourritures ou en se sustentant de souffle, les taoïstes vont se conjoindre au monde sauvage. Végétaux et minéraux, les aliments de substitution des taoïstes s'opposent aux céréales par leur appartenance au monde de la nature brute. Les biographies de saints taoïstes s'étendent avec complaisance sur les anachorètes qui s'alimentent de fleurs de pêcher, d'aiguilles de pin, de jujubes séchés, de fruits de cannelier, de champignons de montagne, d'iris jaunes des marais, de fuling 获苓, huangiing 黄 精 xionghuang 雄 黄, etc. Sauvages, ces nourritures le sont d'une double manière : par les lieux impénétrables, les montagnes inexplorées où elles croissent, mais aussi et surtout par l'effet

25. Sur l'importance d'avoir les intestins libres pour la circulation du souffle et les méthodes qui permettent d'évacuer les excréments par le souffle, Cf. YJQQ 62, Zhuịia $f a$ 諸家氧法 $5 \mathrm{a}, 7 \mathrm{a}, 9 \mathrm{a}, 13 \mathrm{a}-13 \mathrm{~b}, 14 \mathrm{a}$. 
qu'elles produisent sur l'adepte : habituellement leur corps se couvre de poils. Le Baopuzi consacre tout un chapitre à énumérer les exemples d'hommes à qui l'abandon des grains et le choix d'autres nourritures confèrent une apparence animale ${ }^{26}$. A côté des taoïstes velus il existe aussi des taoïstes emplumés : le thème est ancien. Le Huangting jing (wai) (Concordance II, 4-5) dit "Qu'à celui qui nourrit son corps et sait tenir la vacuité pousseront des ailes et il s'élancera dans la nue $\gg^{27}$. Ils acquièrent alors des qualités d'endurance et de vélocité qui les rendent semblables aux animaux ${ }^{28}$. Wo Quan cueillait des simples sur les montagnes et se nourrissait exclusivement de pignons. Il avait de longs poils sur le corps et se déplaçait à la vitesse d'un cheval au galop (Shoushenji 1.1b). Le Shenxian zhuan fourmille d'exemples de saints aussi vites que des chevaux. Nous ne citerons pour mémoire que quelques-uns : Wang Zhen se meut à la vitesse d'un cheval au galop (10.4b); Lü Nüsheng parcourt trois cents lieues par jour et force à la course les chevreuils et les cerfs $(10.5 \mathrm{~b})$. Wang Lie marche comme en volant $(6.2 \mathrm{~b})$. Cette vélocité contraste avec la lenteur des chevaux nourris aux grains. Elle est le signe avant coureur de

26. Han Zong absorbait des iris jaunes; au bout de treize ans son corps se couvrit de poils et il ne ressentit plus la morsure du froid $(11, p, 209)$. Zhao Tuzi, après s'être sustenté de cannelle pendant vingt ans eut les pieds recouverts de poils et put parcourir cinq cents lieues par jour (11. p. 210). Et le même ouvrage citant le Shennong sijing $(11$, p. 183) proclame que l'un des effets des drogues supérieures (shang yoo 上薬) est de faire apparaître des poils ou des plumes sur le corps. Le Shenxian zhuan (1.4a) rapporte un long entretien entre Penzu et une adepte. Pengzu explique à son interlocutrice que les immortels se reconnaissent aux poils qui leur couvrent le corps.

27. Sur le rapport entre taoïstes et oiseaux, cf. notamment le Liexian zhuan où sont cités un certains nombre de taoïstes en relation avec les grues.

28. L'endurance au froid, par exemple, est un des poncifs de la littérature hagiographique taoïste. Le Shenxian zhum donne de multiples exemples de ce type. Liu Gen est nu été comme hiver et de longs poils lui couvrent le corps (3.1b). Li Yiqi ne porte qu'un habit simple en hiver (8.3b). Sun Deng ne revêt qu'un habit de toile en toute saison. En hiver, lorsqu'il fait vraiment froid, il se couvre le corps de ses longs cheveux (6.3b). Le thème sert à marquer l'adaptation du taoiste au monde naturel. 
l'immortalité. Le Zhenzhongji (19a) donne une recette de mica (yunmu 雲母) qui permet après avoir évacué les Trois Vers et si on la prend régulièrement durant trois cents jours - d'aller à la vitesse d'un cheval au galop puis de devenir immorte ${ }^{29}$.

Par ailleurs la diète taoïste s'accompagnait d'exercices gymniques. Le Zhuangzi (Pères : 331) et le Huainanzi (8. p. 224) mentionnent des danses qui consistent à imiter des postures animales 30 . Les Sanguo zhi $(29$, p. 804$)$ et Houhan shu $(82$, p. 2740) attribuent tous deux à Hua Tuo 華 陀 une danse des cinq animaux : danse du tigre, de l'ours, du cerf, du singe et de l'oiseau permettant d'éliminer le souffle des céréales. En la pratiquant, $\mathrm{Wu} \mathrm{Bu}$ 吴普 un disciple de Hua Tuo, était encore jeune à l'âge de 90 ans. Ainsi la gymnastique consistant à imiter des postures animales aboutit au même résultat que l'abstinence des céréales et l'ingestion des drogues ${ }^{31}$. Ecartant les effluves pestilentiels des céréales, elle permet d'échapper à la sénescence. Il y a donc homologie entre abstinence des céréales et apparence animale.

Cet ensauvagement du taoïste se marque aussi dans sa dilection pour les montagnes : l'adepte entreprend de longues courses solitaires dans les monts et les forêts. Il se retire sur des cimes inaccessibles pour cueillir les simples ou les matières minérales dont il s'alimente. C'est sur ces montagnes, parmi les tigres et les rhino-

29. Le Taiqingiing duangufa(5b) indique une recette de huangjing (poligonatum giganteum, var. Thumbergii) supprimant la faim et rendant aussi rapide qu'un cheval au galop.

30. On se rapportera aussi aux représentations des exercices gymniquestrouvées à Mawangdui et dont plusieurs portent des titres d'animaux (Wenwu, 1975, 6).

31. On trouve aussi des allusions à cette danse dans des textes du canon taoiste. Le Yangxing yanming $l u(7 \mathrm{a}-\mathrm{b})$ attribué à Tao Honging 陶弘呆 et le Taishang Laojun yangsheng jue la mentionnent. Dans tous ces ouvrages, elle est recommandée pour éliminer le souffle des céréales. 
céros qu'il va chercher un maître pour s'initier aux pratiques alchimiques. Dans une célèbre polémique avec le poète taoïste Xi Kang, le confucéen Xiang Ziqi 向子 期 ne lui reprochet-il pas de prendre « les monts pour voisins ${ }^{32}$ et le représentant de l'orthodoxie confucéenne ne fait-il pas un peu la même critique à son contradicteur dans un dialogue du Baopuzi $(10$, p. 178) mettant en scène les tenants des deux écoles quand il accuse son adversaire de « vivre avec les renards et d'être le compagnon des singes " 33 ? Tout un chapitre du Baopuzi est d'ailleurs consacré aux moyens d'accéder sans danger à l'univers sauvage des forêts et des montagnes et aux charmes et amulettes qui permetttent de se protéger des génies, démons et bêtes sauvages qui hantent ces contrées ${ }^{34}$.

Conjoint à la nature sauvage sur le triple plan des nourritures, de l'apparence et de l'habitat, le taoïste se trouve encore uni au monde bestial par la folie. Les taoïstes sont dépeints sous les traits d'insensés. On connaît bien le fou de Chu qui railla Confucius ${ }^{35}$. Insensé, tel paraissait aux yeux de ses contemporains le célèbre mage taoïste Dongfang Shuo (Liexian zhuan : 93). Est aussi qualifié de fou (changkuang 猖狂) le sage inspiré, Hongmong 鸿蒙 《Tohu-bohu ». Il marchait à cloche pied et prêcha au malheureux politicien Yun Jiang le retour au chaos (Zhuangzi, Pères, p. 288). Or la folie connote la sauvagerie sur une multiplicité de plans. Le mot qui sert à la désigner, kuang 狂 a des significations multiples et complexes, mais elles traduisent toutes une perte de sens, un

32. Nan yangsheng lun (Lunxun quanji IX, p. 60). Une expression voisine figure dans le Shenxian zhuan (4.4b).

33. Une formule identique est reprise dans le Hunsu yisheng lu (la) : « Etre le compagnon des singes et s'attrouper avec les cerfs \#.

34. Il s'agit du ch. 17 intitulé : dengshe 登涉.

35. Un paragraphe du Liexian zhuan (p. 93) est consacré à un certain Loutong qui n'est autre que le ufou de Chu». 
dérèglement qui provoque une transgression de la norme. Elle est la marque d'une régression vers le chaos, le désordre ou l'état de nature - qui en est l'expression atténuée ${ }^{36}$. Jugée positivement ou négativement, la folie est toujours violation d'une norme. Par là elle précipite celui qui en est saisi dans un univers qui est en marge de la vie sociale ou qui l'ignore totalement : elle met à l'écart, isole. Bref, elle est le signe de la liminalité. Le terme kuangfu 狂夫 " furieux 》 désigne ces rois de perdition, ces monarques dégénérés qui ramenèrent la Chine à un état voisin de la barbarie des premiers âges en introduisant dans leurs palais des fauves ou en laissant s'ébattre des hommes et des femmes nus au milieu de quartiers de viande (Huainanzi, pp. 296, 253) ${ }^{37}$. Ces fous deviennent comme des animaux : «ils ont une conduite de bête et une cupidité de tigre ou de loup » (Xunzi, p. 188). La même expression sert à qualifier les hommes de l'antiquité qui, ne connaissant pas les arts de la civilisation, sont eux aussi comme des insensés : ils ne connaissent ni la droite ni la gauche (Huainanzi, p. 44; Zhuangzi :Pères, p. 275). Symbole de bestialité, la folie peut provoquer des transformations

36. Kuang désigne en effet la perte de la faculté de raisonner et donc de séparer et d'ordonner que produit la colère ou la peur. Dans le Huainanzi $(24$, p. 226) par exemple une grande frayeur peut provoquer une crise de folie. Il désigne aussi la fureur guerrière qui altère la personnalité; le syntagme kuangii 狂疾 apparaît dans le Guoyu (9.p. 175) où il est glosé par 《kuang er yi changxing 狂而易常性》 devenir fou et changer de caractère. Le mot kuang désigne aussi la conduite inconsidérée des princes qui ne respectant plus les autres ne sont plus respectés, ou encore l'aveuglement qui les empêche de reconnaître le vrai talent provoquant l'apparition de signes aberrants (kuang). Par ex : Hanshu : Wuxing zhi, 1342-1343), Houhanshu, Wuxing zhi I : 3265 - Cf. aussi Guanzi, II, 11, p. 175. Troubles de la personnalité, aveuglement du seigneur négligeant ses devoirs, le terme finit par désigner toute chose anormale ou anarchique : les plantes qui ne poussent pas à la saison voulue, le blé qui donne des épis trop hâtifs, de même que la rage dont meurent les chiens dans les années de sécheresse (Soushen ji, 7, p. 122; Huainanzi, $5, p .184)$. Il peut désigner enfin cette attitude presque rituelle derrière laquelle se cache un sage pour échapper à la vindicte d'un prince corrompu et fuir des temps troublés (Wuyue chunqiu, p. 28 et 227; Huainanzi, p. 401).

37. Cf. aussi Diwang shiji, p. 17; Guanzi, II, p. 4 ; Mengzi (Oeuvres 2, p. 551). 
en tigre. Cette idée ne s'exprime pas seulement à travers un folklore tardif ${ }^{38}$, dès le II ${ }^{\mathrm{e}}$ siècle av. J.-C. elle faisait partie des notions communément admises ${ }^{39}$. Les hommes tigres du folklore ancien se rapprochent des chercheurs d'immortalité : la folie qui les frappe avant leur transformation est le signe de leur ensauvagement, tout comme chez les taoïstes elle marque leur volonté de se retrancher de la société. Elle constitue aussi dans l'un et l'autre cas une forme de transe. L'homme tigre n'est plus lui-même, il est possédé par des esprits de la forêt ${ }^{40}$ et les taoïstes sont habiles à faire descendre en eux des esprits ${ }^{41}$.

Si le refús des céréales entraîne les taoïstes dans l'univers sauvage qu'aucun feu n'a cultivé, l'ingestion de drogues spéciales les voue au monde du brûlé. L'un des effets produit par les drogues est de provoquer une chaleur interne : le Baopuzi recommande de se laver les cheveux dans l'eau glacée et de marcher contre le vent. Certaines drogues minérales échauffent à tel point l'organisme que l'on peut se tenir nu en plein hiver et manger de la glace sans éprouver aucune incommodité (Baopuzi 11, p. 201). Ces symptômes évoquent fort ceux qui précèdent la délivrance par le cadavre (shijie $\rho$ 解) : l'adepte avant de se dépouiller de son enveloppe

38. Sur les contes relatant des métamorphoses d'hommes en tigre : Cf.Yiyuan, 5.1a; TPGJ, 426, p. $3468 ; 427$, pp. 3479,3476 , etc.

39. Le Huainanzi fait allusion à la transformation de Gong niu ai en tigre, comme à une anecdote universellement répandue (2. p. 42). L'histoire est longuement commentée aussi par le Lunheng (16. p. 250; 20, p. 316).

40. Cf. par exemple le Yiyuan (8. 5a) où un homme devient possédé car il a reçu l'appel d'un dieu du sol. Toutes les histoires de Changgui 倀鬼 tournent autour de la possession. Et on ne peut manquer de faire le lien entre les histoires de tigne du folklore chinois et celles des minorités de l'Asie du Sud-Est (Cf. D.C. Graham 1954).

41. M. Kaltenmark, 1960, pp. 559 - 588 et Granet, 1953, pp. $245-249$ ont traité de la possession taoïste à époque ancienne. 
corporelle et d'accomplir sa transmutation est saisi d'un feu intérieur qui le dévore comme un brasier. On est obligé d'arroser constamment son corps d'eau froide. Lorsqu'on l'en asperge c'est « comme si l'on répandait de l'eau sur des pierres brûlantes » (Shenxian zhuan, 2. 2a) ${ }^{42}$. Le Shenxian zhuan et le Baopuzi renferment le nom de certaines médecines qui rendent lumineux comme des astres et des recueils de recettes tao istes citent un grand nombre de drogues qui après avoir évacué les Trois Vers rendent resplendissant. Une recette attribuée à Zhou Yishan préconise un régime qui " rend le corps léger et lui fait une auréole ». Comme le note Maspero (1970, p. 370) il s'agit peut-être ici d'une lumière intérieure qui permet à l'adepte de voir à l'intérieur de lui-même. Mais d'autres textes font clairement référence à une émission de lumière externe. Par exemple, Sun Simiao 孫思邀 donne des recettes de drogues qui procurent un teint resplendissant (Maspero, 1971, p. 371). La vertu des drogues se manifeste par la luminosité qu'elles confèrent. Ces efflorescences minérales que sont les agarics des Sept Lumières ou des Neuf Flambeaux provoquent dès qu'on les consomme une bouffée de chaleur dans l'organisme et rendent lumineux. Celui qui s'en nourrit peut voir en pleine nuit ou dans l'obscurité la plus totale (Baopuzi, 11, p. 188). Le mica a les mêmes propriétés : Dong Guoyan fut capable de lire la nuit après en avoir mangé. (Shenxian zhuan, 10. 5a). Un appendice au Hanwudi neizhuan, le Hanwudi waizhuan, insiste lui aussi sur l'embrasement du corps du taoïste : " le souffle rouge qui entre dans la porte céleste et fait le tour du corps à l'intérieur comme à l'extérieur, se transforme en feu dans le cerveau, incendie celui-ci et lui donne

42. Cf. Sur cette question les intéressantes remarques de R. Stein (1972, p. 499). 
même éclat que les flammes d'un brasier ${ }^{43}$. L'absorption des drogues aboutit à une auto-crémation. Aussi n'est-il pas étonnant de voir les taoïstes détourner tout un ensemble de mythes qui ont trait à la maîtrise des arts du feu et aux maîtres de forge pour les utiliser à leurs propres fins ${ }^{44}$. L'une des manières de se rendre aux Kunlun, sorte de paradis des immortels, est de se consumer sur un bûcher. La crémation apparaît donc comme un équivalent symbo lique de l'ingestion des drogues minérales : le Baopuzi $(11$, p. 201) nous dit que Chisong zi, Sapin rouge, serait devenu capable de monter et de descendre au gré de la fumée après avoir avalé du jade liquide ${ }^{45}$. Le même Chisong zi selon le Liexian zhuan p. 36

43. Maspero, 1971, p. 523. Maspero a traduit tout un passage d'un ouvrage taoiste des Tang : "Livres nouveaux et anciens de l'absorption des souffles du Maître Yangling 》, Yangling xiansheng ji xinjiu fuqijing 延陵先生集新鹪服羨经 Celui-ci cite une formule de Juanzi 滑子 préconisant de se représenter le corps comme fondu par le feu et devenu braises (p. 520).

44. M. Kaltenmark (Lie Sien Tchouan) a noté la parenté entre les légendes sur les maîtres des techniques du feu et les biographies taoïstes du Liexian zhuan. La crémation sur un bûcher serait à rapprocher des anciennes pratiques pour faire venir la pluie; on exposait au soleil ou faisait brûler une sorcière. Ces vieux thèmes ont été réutilisés pour symboliser la communion avec le monde divin et d'anciens mythes sur les sorciers maîtres de pluie ont été réinterprétés comme des légendes d'immortels accédant à la divinité en se vouant au monde du brûlé. Un processus analogue a joué sans doute dans l'autocrémation bouddhique qui fit fureur à l'époque des Six Dynastie (Cf. Gernet, 1960. pp. 527-558). M. K.M. Schipper, 1982, assimile la crémation des écritures et l'involution taoïste qui, après avoir créé un enfançon d'immortalité dans sa matrice, le détruit pour se fondre dans le chaos, à une oblation (pp. 122 sq; 207-208). Les anciennes pratiques sacrificielles où la victime était consumée sur un bûcher seraient alors à la base de ces conceptions. Notons qu'il serait plus exact de parler de contre sacrifice ou d'anti-sacrifice, car au rebours de ce qui se passe dans la pratique sacrificielle normale où il s'agit de marquer dans la communion même qui unit pour un temps dieux et hommes leur écart, la crémation taoïste abolit la distance entre les deux univers.

45. Il ajoute que le jade provoque une chaleur intense. 
se serait livré à la flamme ardente d'un bûcher pour enseigner son art à Shennong ${ }^{46}$. D'autres encore auraient obtenu l'immortalité par ce moyen : Ningfeng zi, Shimen, Jiezi Tui, Xiao Fu, etc ${ }^{47}$.

Cette crémation, qu'elle soit obtenue par la flamme ardente provoquée par des drogues ou par la seule circulation du souffle, confère des pouvoirs magiques sur lesquels les biographies taoïstes s'étendent avec complaisance : l'immortel entre dans l'eau sans se mouiller, traverse les flammes sans se brûler, affronte les armes sans être blessé; il peut faire descendre des êtres transcendants, voler sur des nuages, se tranformer en tous les êtres de la création, etc. Les lieux qu'il hante, les montagnes impénétrables où il s'ébat le mettent de plain-pied avec la divinité. Il peut, enfin, comme les déesses et les dieux faire surgir des «banquets de voyage " (xingchu 行剭 ) où sont servis des vins provenant des cuisines célestes ${ }^{58}$.

Les taoïstes tournent la condition "moyenne » de l'humanité " cultivée » par le bas et par le haut. Les drogues en effet apparaissent comme symétriques de l'abstinence des céréales. Mais tandis que cette dernière permet d'échapper à la condition humaine en se plaçant en deça, en se conjoignant au monde sauvage, celleslà, la tournent par le haut, se plaçant au-delà, fournissant l'accès au divin, domaine du brûlé 49

46. Pour une autre version, Cf. Shoushen ji, 1. 1a.

47. Cf. Liexian zhuan pp. $36,43,74,86)$ et Shoushenji, 1. 1a, 2a, 3 b.

48. Sur les xingchu, Cf. infra.

49. Dans la pratique sacrificielle en effet, le brûlé (ainsi que les aromates) est consacré au dieu suprême (Shangdi 上帝) puisqu'au sacrifice dans la banlieu sud, la victime est entièrement brûlée sur un bûcher et le dieu se nourrit des fumets sacrificiels : "Nous remplissons d'offrandes les coupes en bois; les coupes, en bois, les coupes en terre. Dès que le parfum est monté le Seigneur d'en Haut se met à manger "Maspero 1971, p. 23. 
Brûlé-divin est symétrique au couple cru-animal (dont le mode extrême est l'anthropophagie) ${ }^{50}$ par rapport à une ligne médiane constituée par le binome humain-céréales.

Chacune des trois espèces (hommes, animaux, dieux) entre lesquelles se répartissent les êtres qui peuplent l'univers est donc caractérisée par un type de nourriture. Mais les deux poles brûlé/ divin, cru/animal viennent se confondre pour s'opposer au couple cuit/humain. Cette structure trouve sa traduction géographique dans une tradition mythique rapportée par $\mathrm{Ma}$ Duanlin à propos des Monts Limu : sur les premiers contreforts de la montagne habitent les Shuli 熟黎, les 《Li cuits》, ceux qui ont été déjà transformés par le feu de la civilisation; plus haut, dans les lieux escarpés et impénétrables, vivent les Shengli 生黎 -, les «Li crus》, qui n'ont connu aucun feu civilisateur; au sommet du Mont Limu, dans un sublime isolement se tiennent les immortels, coupés des hommes par des précipices gardés par des tigres (Wenxian tongkao, 331, p. 2599B).

A ces trois modalités culinaires; cru, cuit, brûlé, correspondant à chacune des trois espèces, doit s'ajouter une quatrième catégorie, le pourri. Il est assimilé par la pensée taoïste à l'une des formes extrêmes de la cuisson et forme couple avec le cuit. En tenant compte de ces quatre termes et en les distribuant selon les

50. Des Rotours a étudié le problème de l'anthropophagie en Chine ancienne d'une façon thématique. L'expression ren xiangshi 人相食 que l'on retrouve à maintes reprises dans les annales et les classiques renvoie moins à une réalité historique qu'elle ne connote la sauvagerie d'un monde en proie au chaos et à la barbarie des premiers âges. Que le cannibalisme soit la forme extrême de l'omophagie apparaît très clairement dans le riche folklore sur les hommes-tigres. La manducation des viandes crues suffit à provoquer des métamorphoses en tigre (Cf. TPGJ, 432, 3506; 430, 3495 et Yiyuan, 8.5b; Shuyïi in Guxiaoshuo gouchen, p. 289). Or seuls les hommes-tigres sont cannibales (Cf. Shuyijing, 1.3b). L'omophagie est l'équivalent humain de l'anthropophagie animale. 
catégories d'êtres, on obtiendrait le tableau suivant :

Brûlé

cuit

pourri

cru dieux

hommes

vers des céréales

nature immortalité

vie brève

longue vie

Ce tableau ne correspond pas au modèle que Lévi-Strauss a cru dégager de l'analyse des mythes américains. Selon son schéma, les couples opposés devraient être : brûlé/pourri et cuit/cru. Le modèle induit par le grand anthropologue est sans doute trop rigide, chaque groupe social jouant avec le système et le détournant à ses propres fins. Si l'on devait élaborer un diagramme à partir des conceptions chinoises traditionnelles, on obtiendrait sans doute un tout autre tableau : tout au moins, le pourri deviendrait une des modalité extrêmes du cru. Un passage du Gushi kao (p. 363) retraçant la vie de l'humanité primitive insiste sur l'effet nocif des nourritures crues sur les intestins des hommes de l'antiquité51. Au niveau du vocabulaire, la même assimilation se retrouve dans le mot xing 腥 : il désigne tout à la fois la viande crue, l'odeur du poisson frais, mais aussi les effluves désagréables de ce qui est passé, de la viande pourrie ou du poisson saur. ${ }^{52}$.

Pour mieux asseoir leur contre-système, les taoïstes ont tracé tout un réseau de correspondances entre les deux termes symétriques, si bien que faire la bête permet dans un même mouvement

51. C'est l'un des thèmes constants de la littérature " orthodoxe » consacrée à la vie des hommes des premiers âges. Cf. Baopuzi (wai), p. 777, Fengsu Tongyi, p. 3, Liji (mémoires : 1. 504), Han Fei (1040), Lunheng, p. 291, Xinyu, 1. 2b. etc.

52. Dans toute la littérature traitant des âges primordiaux, les effluves méphitiques de la viande et du poisson crus " pourrissent " les viscères. Par exemple dans le Laojun kaitian jing on trouve l'expression : xingseo chousi 腥臊奥檅. 
d'accéder au divin et réciproquement, accéder au même statut que les dieux, c'est se mettre de plain-pied avec les betes féroces.

Il y a un va et vient constant entre nature et surnature, entre bêtes et dieux. Nous avons vu que la fuite dans les montagnes était l'un des thèmes favoris des biographies sur les saints taoïstes et qu'elle traduisait leur ensauvagement. Or l'ascension des montagnes équivaut à une montée au ciel. Le Huainanzi (4, p. 124) ${ }^{53}$ cite les noms de cimes qui confèrent l'immortalité à celui qui les gravit. Les taoïstes se plaisent à vivre dans des grottes : celles-ci sont des ciels et les gouttes qui tombent des cavernes pour former des stalagtites sont parmi les plus efficaces des médecines pour obtenir l'immortalité ${ }^{44}$.

Un des meilleurs moyens de s'ensauvager est d'exécuter des danses animales ou d'imiter la respiration des tortues ou des serpents. Ces pratiques confèrent une longévité égale à celle des dieux. (Bowuzhi, 2, p. 15). Car les animaux sauvages eux-mêmes vivent presque éternellement : les singes, au terme d'une série de transformations, atteignent quelque deux mille ans d'âge; les tigres, les cerfs, les lièvres vivent mille ans; les renards, les loups, les chats sauvages traversent les siècles; les licornes, certains chevaux ne meurent qu'après plusieurs centaines d'années; il n'est jusqu'aux rats qui ne jouissent d'une longévité exceptionnelle. Leur grand âge leur confère des pouvoirs qui n'ont rien à envier à ceux des adeptes taoïstes. Les tigres peuvent se transformer en tous les êtres de la création, les renards savent se métamorphoser en homme, les vieux

53. Cf. aussi Lunheng, pp. 107-108 où l'excursion sur les monts Kunlun équivaut à une montée au ciel.

54. Sur les taoïstes qui vivent dans des grottes, Cf. Shenxian zhuan $3.1 \mathrm{~b}, 3 \mathrm{~b}, 4 \mathrm{a}$, 5.3a, 6.3b. Sur le rapport entre grottes et ciels, voir Lie Sien Tchouan, p. 36, n. Le Baopuzi mentionne parmi les drogues d'immortalité les stalagtites (11, p. 189). 
rats prévoient l'avenir, etc. ${ }^{55}$. La préface du Hunsu Yisheng lu (2a-2b) oppose la longévité des animaux sauvages et des premiers hommes à la brièveté de l'existence des hommes civilisés.

Cette ambivalence, ce renversement constant, se retrouve dans les conceptions qui tournent autour des "banquets de voyage" (xingchu) ou des 《banquets célestes》(tianchu 天厚 I. Ceux-ci désignent les repas communiels auxquels participent les divinités présentes dans le corps de l'adepte lors des repas maigres et des banquets collectifs des taoïstes. Le terme "banquets de royage désigne aussi les techniques individuelles de salut. Ce sont alors des repas célestes (tianchu) que peut faire descendre l'initié après l'ingestion de certaines drogues. Ils sont autant le signe de l'immortalité que le moyen par lequel il la renouvelle ${ }^{56}$.

Dans un grand nombre de légendes taoïstes ils apparaissent comme un don en retour d'une divinité à l'adepte. C'est ainsi que Magu après avoir reçu un repas rituel consistant en alcool fait descendre des "banquets de voyage » constitués principalement de vin, de viande séchée, de jujubes et surtout de fruits (Shenxian zhuan :2. p. 2b, 7. p. 2a).

Dans la légende de l'Empereur $\mathrm{Wu}$ des $\mathrm{Han}^{57}$, l'adepte offre une collation de viande séchée, de jujubes et d'alcool. Les déesses

55. Baopuzi : 3, pp. 36-38. Cf. aussi TPGJ, 426, p. 3466 .

56. Sur cette question des « banquets de voyage » et des cuisines célestes on se reportera aux pages pénétrantes de Stein (1971, p. 489) et (1972, p. 492).

57. Sur la visite de la Reine mère d'Occident (Xiwang $m u$ ) au roi Wu des Han, voir l'étude désormais classique de K.M. Schipper, 1965.

58. On notera que dans l'une le repas offert à l'adepte est appelé «banquet céleste.» tandis que dans l'autre c'est l'expression « banquet de voyage 》 qui est utilisée. Les deux expression semblent interchangeables. 
après avoir offert en contrepartie un repas de nourritures divines (tianchu) l'instruisent des drogues et des talismans qui rendent immortels. La descente de Magu et la visite de la Xiwang mu 西 王 $母$ ont bien des traits en commun ${ }^{58}$.

Or l'expression «banquets de voyage » (xingchu) synonyme de tianchu ( nécessitant pas de longs préparatifs et composés d'aliments de " crise ${ }^{59}$. Ils servaient aux laïques dans les périodes où il s'avérait impossible de se livrer à des préparations culinaires. Ces repas étaient présentés lors de la fête du manger-froid (hanshi 寒食) correspondant à l'antique sortie des feux qui précédait une extinction totale des foumeaux proclamée par un fonctionnaire au moyen d'un claquoir ${ }^{60}$. Ce temps de latence traduisait une période de sauvagerie, de vacance du feu de cuisine. Dès lors, la fête du manger-froid n'est qu'une forme atténuée du manger-cru. Les cuisines des dieux sont des cuisines de sauvagerie.

Cette polarité se vérifie à l'intérieur même de certaines nourritures qui composent l'essentiel de ces pique-niques divins. Bien que bannie de certains rituels ${ }^{61}$ la pêche n'en est pas moins considérée dans les contes taoïsants et les biographies de saint taoïstes comme le grand fruit des immortels. Le Taishang Dongyuan shenzhou jing

59. On les retrouve en effet dans les rations des soldats en campagne (Cf. Stein. 1972, pp. 492-495).

60. Lushi, Qiangii, 5.4b, fahui, 1.8a-10b; Zhouli (Tcheouti) : IV. p. 67; XXX, p. 194; D. Bodde, pp. 296, 299-302. Sur le problème général de l'extinction du foyer et de son rapport avec le retour à sa sauvagerie, cf. Lévi-Strauss 1964, pp. 304-305; 1967, pp. 355-357.

61. Cf. K.M. Schipper 1965. p. 23. Il semblerait cependant que cette interdiction ne soit pas aussi formelle qu'il ne le laisse entendre. Elle n'est déconseillée qu'avec certaines drogues (Cf. Zhengzhong ji,26a; Taiqinging duangu fa, 5a) 
(1. 1a-b) fait allusion à un immense pêcher qui se dresse sur la montagne Yujing 玉 京 et dont le fruit unique ne vient à maturité qu'au bout de milliers d'années. Il ôte la faim à celui qui le consomme durant trois mille ans. Symbole de longévité, la pêche constitue le mets principal dont la Xiwang $m u$ régale ses hôtes pour qu'ils renouvellent leur immortalité (Schipper 1965, p. 53). Un certain nombre d'immortels en font leur nourriture quotidienne ${ }^{62}$. Avant de disparaître au ciel dans une apothéose, Zhang Daoling, le fondateur du courant des Maîtres célestes, procéda à une distribution de pêches entre ses trois mille disciples (Shenxian zhuan 4. 4b). Zhang Daoling, à l'instar de la Xiwang $m u$, autre pourvoyeuse de pêches, est détenteur d'un élixir d'immortalité63. Un autre conte narre comment tous ceux qui suivirent Ge You sur le mont Wei où poussait une forêt de pêchers ne revinrent jamais. Un adage dit depuis lors : «même si les pêches de la montagne Wei ne suffisent pas à procurer l'immortalité, elles rendent courageux et éminent $»($ Soushen $j i, 1$. 3a). C'est encore une pêche qui fut remise à la future Guanyin pour qu'elle ne souffre ni de la faim ni de la soif durant son voyage vers l'île de Puto où elle allait devenir bouddha (De Groot $1888: 14^{\mathrm{e}}$ jour du $2^{\mathrm{e}}$ mois).

Fruit divin, la pêche ainsi que le pêcher tient par tout un autre côté au monde sauvage. Les images divines auxquelles elle est associée ont une autre face, bestiale celle-là. La Xiwang $m u$ avant d'être une grande déesse taoïste fut un monstre à queue de

62. Dong Zigong se nourrit exclusivement de pêches et d'eau pure (Shenxian zhuan, 10: 5a), Lü Nüsheng, quant à lui se sustente de fleurs de pêchers, tout comme Shimen (Shenxian zhuan : 10.5b ; Soushenji : 1.2a). L'immortelle He Xiangu aurait reçu l'immortalité par la manducation d'une pêche (Doré : II, pp. 477478. Cf. aussi De Groot 1910 : IV, pp. 297-324.

63. Doré : II, p. 527. Pour la Xiwang $m u$ détentrice de l'élixir d'immortalité, cf. Huainanzi, 6, p. 209. 
léopard, habitant le Kunlun et nourrie de chair crue par trois corbeaux ${ }^{64}$. Le retournement n'est pas fortuit : Zhang Daoling est lui aussi lié aux fauves. On le représente chevauchant un tigre dans l'iconographie populaire. S'il est vrai que cela est le cas pour quelques autres génies ou dieux ${ }^{65}$, sa fonction de pourfendeur de démons donne à ce détail une tout autre signification. En effet, on suspendait des images de Zhang Daoling sur les vantaux des portes lors des fêtes de nouvel an pour écarter des maisons les fantômes et les maladies. De la même façon on clouait des effigies de tigres au-dessus des portes pour se protéger des influences malignes ${ }^{66}$. Pêches et pêchers sont liés au tigre, cet animal du monde sauvage : ce sont comme lui de puissants démonifuges. Une antique légende raconte comment deux génies qui se trouvent près du pêcher Dushuo donnaient en pâture aux tigres les démons malfaisants ${ }^{67}$. La légende du grand pêcher de la montagne Yujing ne manque d'ailleurs pas d'évoquer ses pouvoirs apotrophaïques. De même que les tigres hantent les cimes escarpées et les gorges profondes, les pêchers croissent près des falaises ou sur des pics inaccessibles. Et si la pêche est le fruit des divinités taoïstes, elle est dans les contes du folklore moderne une nourriture dont les tigres sont gourmands (B. Hensman, p. 23). Si bien que l'on peut douter que ce soit pure coincidence si la Guanyin chevauchait un tigre lorsqu'elle reçut le fruit précieux...

Il y a dans les pratiques alimentaires des taoïstes un mouvement de balancier, une oscillation constante entre des termes opposés et

64. Shanghai jing : $16.76 \mathrm{~b}$ et $12.54 \mathrm{~b}$.

65. Doré : II, p. 702 donne un certain nombre d'immortels chevauchant des tigres.

66. Sur ces pratiques, Cf. Granet 1959, I, p. 299. Les amulettes sculptées dans des noyaux de pêche constituent une excellente antidote contre les fièvres qui attaquent les petits enfants. Les images de tigre recèlent la même efficacité (Doré : V, p. 504).

67. Granet 1959 : I, 303 analyse longuement le mythe. Cf. aussi sur cette question l'excellente étude de D. Bodde 1975, pp. 127-138. 
contradictoires : le cru et le brûlé, le bestial et le divin. Récusant les céréales, les chercheurs d'immortalité se conjoignent à la nature sauvage tout en accédant au divin. La diététique taoïste n'est pas plus une collection de recettes de bonne femme qu'une hygiène proto-scientifique. Elle s'interprète dans un système global de refus d'une orthodoxie : elle n'a de sens que par référence à une réflexion sur l'Age d'Or et l'évolution de l'humanité.

\section{III - La Divine Confusion}

Le sage taoïste est celui qui, sachant faire corps avec le Dao (tidao 骨量道) ou tenir l'Un (shouyi 守一) retourne à l'indistinction primordiale. Le Dao, en effet, vient se confondre dans la méthaphysique taoïste avec un ensemble de notions ou d'expressions qui en paraissent si proches qu'elles en deviennent synonymes: toutes sont empruntées au vocabulaire du chaos. Dans Huainanzi $(1$, p. 1), le Dao est vu comme quelque chose de trouble, d'indistinct (hunhunbobo 混混泊泊). Dans le Wenzi (1,p. 1) il est à plusieurs reprises qualifié de 《sans forme» (wuxing 刑形). Dans le Huainanzi le sans forme est un grand chaos où toutes les choses viennent se fondre en une (suo wei wuxing zhe yi wei ye... dahun er wei yi ye 所謂無形者一之潘也... 大渾而 桨 一葉 ) (1.p. 21). L'expression revient constamment sous le pinceau des taoïstes pour désigner l'étape première de la formation de l'univers. Cherchant à définir le Dao, le Laozi dit : « le regardant, on ne le voit pas : on le nomme invisible. L'écoutant, on ne l'entend pas : on le nomme l'inaudible. Le touchant, on ne le sent pas : on le nomme l'impalpable. Ces trois états se fondent en un (hun er wei yi 渾而洺一)》(Pères, p. 29). C'est en termes identiques qu'est décrit dans le Huainanzi (1.p.51) cet état de l'univers où seul existait le néant : « Le regardant on ne le voit pas, l'écoutant on ne l'entend pas, cherchant à l'étreindre on ne 
peut le saisir ${ }^{68}$. Chez Zhuangzi le terme " néant 》 désigne à la fois le magma indifférencié qui a produit tous les êtres et la perfection du Dao qui produit l'univers à tous les instants. Au chapitre 12, quand il décrit l'origine de l'univers, il qualifie l'Un, premier être créé, de non-sensible, de qui-n'a-pas-ncore-de-forme (weixing 未 形 ). Au chapitre 22, c'est le Principe, le Dao lui-même, qui est décrit comme sans forme (wuxing 無形) (Pères, 298; 396) et les mêmes expressions reviennent chez Laozi (Pères, p. 30) pour dépeindre le Dao ou le chaos, ou plutôt, cet objet indéfinissable qui existait avant toute chose.

Le sage qui a su appréhender l'unité du Dao retourne à l'indistinction primitive. Il se fait lui-même chaos. Les évocations du zhenren 真 人 chez Laozi comportent des expressions empruntées au champ sémantique de l'indistinction : "Il (le sage) est rustique comme une pièce de bois brut (dun 沌) ... Il est confus (hun 渾) comme l'eau boueuse "(Pères, p. 33 69. Les mots hun et dun utilisés ici séparément, forment en composition un syntagme qui désigne cette entité primordiale sans haut ni bas, sans forme ni direction : le chaos. Un autre passage du Laozi est encore plus explicite : "Celui qui obstrue toute ouverture, ferme sa porte, émousse son tranchant, dénoue tout écheveau, fusionne toute lumière, unifie toute poussière atteint l'obscure identité (shi wei xuantong 是謂玄同) (Pères, p. 51), formule reprise par le Wenzi $(1$, p. 3) « Aux yeux du sage, indifférent au plaisir et à la

68. Cet objet indéfinissable car indéfini qui est à l'origine de la création est désigné dans le Laozi par la périphrase : «la mère de l'univers 》(Pères : 36). Dans le Huainanzi ce qui existait avant que l'être et le nonêtre n'existassent est « le creuset des dix mille êtres".

69. Dans les textes tao istes la confusion est souvent associée à cette bêtise qui est en réalité l'intelligence suprême de celui qui fait corps avec le Dao. Chez Zhuangzi (Pères, p. 298) on trouve la formule * Se trouver en union si étroite (avec le Dao) qu'on est comme abruti et confus 》, (Qi he minmin ru yu ru hun 其合涽绵若患若昏). 
douleur, à la joie comme à la peine, toutes les choses se dissolvent en une obscure identité ».

En se faisant trouble et confusion, le sage revient à ce stade de la création, ou plutôt de l'incréation où le monde était une sorte de tohu-bohu, de magma sans haut ni bas, sans proche ni lointain. Zhuangzi recommande de se fondre dans l'origine du monde (tong yuchu同於初) ou de s'unir au principe de toutes les créations》 (yi tong hu wu suo zao' 以通乎物所造) (Pères, p. 298). Ailleurs encore, il fait dire au sage Hongmong «Dépouillez votre personnalité, renoncez à l'usage de vos sens, oubliez les relations et les contingences pour fusionner avec le grand Tout (datong hu mingxing 大同乎冥涬)... Celui qui aura passé sa vie dans la confusion (hunhundundun 渾渾沌沌) aura suivi sa nature " (Pères, p. 290). Le nom même du personnage n'est pas innocent. Dans le Huainanzi (7, p. 211) on trouve l'expression hongmong hongtong 项濛鳰洞 pour dépeindre la confusion du chaos primordial 70 . Comme très souvent chez Zhuangzi certains personnages ne sont que des notions abstraites dont les noms annoncent et résument les discours.

Revenir à l'indistinction, c'est aussi retourner aux premiers temps de l'humanité, pendant social du chaos cosmique, que les arts de la civilisation ont abolis de même que l'indistinction a cessé d'être quand le ciel, la terre, puis les saisons, les étoiles, taillant des chemins dans la chair vive du chaos, en firent un espace orienté, semé de signes et de repères 71 . C'est cet assassinat que traduit la

70. Le terme mingxing 冥洋, traduit par le 《grand tout» évoque un espace vague et indistinct. Il s'écrit parfois aussi 涬冥 et a le sens de vaste et de confus. Dans le Huainanzi (8. p. 253) il dépeint l'inondation qui recouvre tout. Dans le Lunheng (11, p. 166) il évoque l'état d'indistinction qui régnait avant que le ciel et la terre ne fussent séparés (minxing monghong, qi wei fen zhi lei ye 溟洋濛澒，氧末分之颣也了。

71. Sur les temps primordiaux, Cf. J. Lévi, 1977. pp. 77-78. 
belle métaphore de l'outre chaos, tuée par la sollicitude du roi de la mer du sud, Emporté, et celui de la mer du nord, Etourdi, (Zhuangzi, Pères, . p. 269). Le Baopuzi lui-même ne compare-til pas la vie des premiers âges au chaos qui précéda l'émergence du cosmos organisé ? (waipian, p. 777).

A la confusion du chaos primordial répond donc l'indistinction des premiers âges : "Les hommes de l'antiquité vivaient dans l'indistinction" (gu zhi ren zai hunmang zhi zhong 古之人在 混芝之中) constate le Zhuangzi (Pères, p. 333) et le Huainanzi p. 59 reprend : " à cette époque régnait l'indistinction 》 (hunhun cangcang 渾渾莟腒) et la simplicité de la communauté primitive ne s'était pas disloquée ». Si la formule xuantong 玄同 évoque l'état extatique du sage, indifférent au monde, ou bien l'aspect de l'univers avant la formation du ciel et de la terre, elle qualifie aussi la société des premiers hommes à laquelle les taoïstes aspirent à retourner : "Flétrissez la conduite des légistes comme Zeng Cheng et She Qiu. Baillonnez Yang Zhou et Mei Di, jetez aux orties la charité et l'équité, et règnera à nouveau dans le monde l'obscure identité (xuantong) »(Zhuangzi : Pères, p. 278). Ce retour à l'état de nature ne serait rien d'autre que la doctrine de Chaos, révèle Confucius à un de ses disciples qui s'est laissé séduire par les doctrines taoïstes ${ }^{72}$. Le Baopuzi reprend l'expression pour résumer la perfection des premiers âges où il n'y avait ni pouvoir, ni intérêt, où malheurs et troubles étaient inconnus, où l'on ignorait l'usage des armes et ne bâtissait ni murailles ni fossés (waipian) : 775).

72. Zhuangzi (Pères : 303) : "Cet homme prétend faussement appliquer l'art de Chaos (Hundunshi zhi shu 渾沌氏之術). S'il était un homme qui fût vraiment simple et pratiquant le non agir, fût retourné à l'antique rusticité, qui suivit sa nature tout en vivant dans son siècle, t'aurait-il fait une telle impression ? Comment nous deux pouvons-nous savoir quelque chose de l'art de Monsieur Chaos! " (que Hundunshi zhi shu, yu yu ru, he zu yi shi zhi zai 且渾沌氏之術, 予具汝何足从諳之哉. 
Xuantong, hunming, hunhun cangcang, etc. ${ }^{73}$ autant de formules qui définissant le champ sémantique de l'indistinct, s'appliquent d'une manière ou d'une autre au Grand tout indifférencié, au chaos, manifestation la plus achevée du Dao. Les hommes de l'antiquité vivaient dans la confusion : "Ils ne savaient pas reconnaître leur droite de leur gauche »(Huainanzi, 2, p. 44): « ils restaient dans leurs maisons sans savoir ce qu'ils faisaient; ils sortaient sans savoir où ils allaient 》(Zhuangzi : Pères, p. 275; Huainanzi, 6, p. 202). La description des hommes primitifs ressemble à tel point à celle des sages taoïstes qu'il est parfois difficile de savoir s'il s'agit des uns ou des autres. Mais peut-être la question est-elle absurde : " hommes-réalisés » et primitifs ne font qu'un ; tout au plus peut-on dire que les uns vivent dans le chaos et que les autres y sont retournés. Au chapitre 23 du Zhuangzi, Laozi explique à un néophyte qui l'interroge sur les recettes pour prolonger la vie qu'il faut retourner à l'état du nourrisson « qui ne sait où il va quand il se met en marche, et reste assis sans savoir ce qu'il fait »(Pères, p. 406).

Ce chaos, cette confusion de la vie des premiers âges que les taoïstes appellent de leurs vœux se traduit par une promiscuité généralisée : "Aux tout premiers temps les hommes vivaient avec les élans et les cerfs »(Liezi : Pères, p. 101; Zhuangzi : Pères,

73. On trouve encore le terme hunmang 渾茫 dans le Zhuangzi (Pères, p. 333). Hunming 混冥 est employé par le Huainanzi (8. p. 246; 2, p. 44). « Les hommes de l'antiquité étaient dans un état de confusion》 (you zai hunming zhi zhong 猶在混 冥之中). Ces expressions reviennent dans le Wenzi $(3$, p. 25) pour décrire le chaos ou le Dao : "Quand le ciel et la terre n'avaient pas encore pris forme, obscurs et troublés (yooyao ming ming 窈妿冥冥), ils étaient mêlés l'un à l'autre et ne formaient qu'un (hun er wei yi 浑而㢁 -)》ou encore 《le Dao est le creuset des dix-mille êtres. Sans forme, de toute éternité, silencieux, jamais il ne se meut, il est trouble et confus (datong hunming 大通湜冥)》(Wenzi， 1， p. 3). Voir aussi Huainanzi, 2, pp. 40,45 . 
p. 275, 469; Xinyu, 1.2a). On pouvait tirer la queue des tigres, marcher sur les serpents, regarder dans le nid des pies (Zhuangzi Pères, 274; Huainanzi, p. 251). Cette familiarité avec le monde animal se traduit par un double habitat qui les conjoint à la fois aux oiseaux et aux quadrupèdes. Ils gîtaient dans les cavernes en hiver et perchaient dans les nids en été (Zhuangzi : Pères, , 469; Liji (Mémoires sur les bienséances : t. 1, VII, p. 504) ${ }^{74}$ montrant ainsi leur accord avec l'ensemble du règne animal. Cette parenté avec le monde sauvage est le signe d'une harmonie avec le divin. Car si la confusion rapproche les hommes des animaux elle les accorde aux dieux. De même que l'habitat des barbares que n'a policés aucun art débouche directement sur les contrées des immortels, les temps primordiaux que les confucéens se plaisent à décrire comme des temps de famine, de fureur et de sauvagerie étaient pour les taoïstes un Eden où tous les êtres vivaient en communion. L'homme y était le commensal tant des dieux que des animaux ${ }^{75}$. Zhuangzi loue en ces termes cette époque idyllique : "Les esprits et les dieux se montraient bienveillants et aucun être ne souffrait de mort prématurée »(Pères, p. 333). Douceur du climat et régularité du cycle naturel marquent assez le caractère proprement divin de ces temps bénis: "Il n'y avait pas de miasmes délétères et les étoiles dispensaient une lumière bénéfique »(Huainanzi, 1, p. 44).

74. Cf. aussi Baopuzi (waipian, p. 777). Nous avons vu plus haut que la folie était une des marques du retour à l'animalité. Or les primitifs sont dépeints comme des fous (changkuang 猖狂) (Huainanzi, p. 44). Hongmong, le sage Tohu-bohu qui prêcha le retour au chaos à Yun Jiang était lui aussi un fou (changkuang) marchant à cloche pied (Zhuangzi : Pères, p. 288). Ailleurs (Pères, 371), il évoque les barbares du sud. Ils sont primitifs et rustiques et se conduisent comme des fous (changkuang wangxing ).

75. «Les hommes sages de l'antiquité se réunissaient avec les dieux et les esprits. Ils communiquaient avec les peuples de toutes les régions de l'univers. Ils n'étaient jusqu'aux insectes avec lesquels il ne vivaient unis 》(Liezi, Pères, p. 101). 
Un passage du Gushi kao, qui retrace la vie de l'humanité primitive dont les intestins sont durement malmenés par l'ingestion des viandes crues, s'ouvre sur cette étrange formule : « Les hommes de l'antiquité aspiraient la rosée » (TPYL, p. 361). L'expression est à rapprocher des locutions employées tant par les taoïstes pour décrire les paradis des immortels que par des confucéens pour magnifier les règnes des saints rois ${ }^{76}$.

Mais si dans le Gushi kao l'évocation de la barbarie des temps primordiaux appelle une expression riche d'implications idylliques, celle-ci suscite à son tour la vision d'un univers sauvage : le Baihutong $(1$, p. 19) qui, retraçant la vie merveilleuse des hommes sous le règne d'antiques souverains, emploie le même stéréotype, ne peut s'empêcher de les dépeindre sous les traits de barbares vivant comme des animaux. Mode de vie contradictoire que le texte résume en une formule : "Ils n'avaient pas de demeure mais étaient en accord avec l'efficace magique du ciel et de la terre ».

La forme animale est le signe du divin : les sages de l'antiquité avaient une apparence qui n'était pas humaine. C'est du moins ce que laisse entendre un passage du Liezi(Pères, p. 101) : Fuxi, Shennong, Yu le Grand, en dépit de leurs traits animaux furent de grands sages : Huang di enrôla dans son armée des bêtes sauvages et Kui, le maître de musique du grand Yao, sut faire danser les cent animaux au son des pierres sonores. Et l'auteur de conclure que dans les tout premiers âges, les hommes et les animaux

76. Le Shanhaijing $(16 ; 74 \mathrm{~b})$ fait allusion à un pays de cocagne situé à l'ouest de la redoutable Xiwang $m u$, divinité mi-humaine, mi-tigre, où les habitants se sustentent de rosée douce. Le Liezi (Pères, p. 83) et le Zhuangzi (Pères, p. 211) mentionnent tous les deux des immortels qui vivent sur une île paradisiaque « aspirant le vent et buvant la rosée ». Rosée grasse ou rosée douce (ganlu 甘露) ou encore sources de vin sont des prodiges qui dans l'imagerie édifiante des lettrés sanctionnent le gouvernement parfait des Rois Sages, parangons de toutes les vertus confucéennes. 
voyageaient ensemble ; seuls de nos jours les barbares possèdent encore le don de vivre en bonne intelligence avec le monde sauvage. Une cosmogonie taoïste tardive se montre fidèle continuatrice de cette tradition. Elle peint les hommes de l'antiquité sous des traits animaux : certains étaient affublés d'une tête de bœuf, d'autres avaient un corps de serpent que surmontait une tête humaine, mais tous " se nourrissaient de souffle primordial et buvaient l'harmonie » vivant presque éternellement ('Xuanjin yuanzhi fahui, $11 a)^{77}$.

Le taoïsme philosophique a les mêmes implications, obéit aux mêmes schémas que les pratiques de longue vie; il ne peut y avoir d'accession au divin, de transcendance, que dans un retour à l'animalité car elle en est le négatif, tout comme la sauvagerie n'est que l'autre face de l'Age d'Or. Le retour au chaos est donc symétrique du refus des nourritures céréalières. Faire corps avec le Dao, ce n'est rien d'autre que retourner à ce stade primordial où l'on était à la fois homme et bête et donc Dieu.

La recherche de la longue vie s'inscrit dans une réflexion globale sur le devenir humain ; elle se situe aussi dans un ensemble compact de mythes et de récits qui traitent de la perte de l'Age d'Or par suite de l'apparition du travail.

Le mythe de Chong et $\mathrm{Li}$, narrant la séparation des hommes et des dieux, par le régulateur du Sud (Chong) qui réunit les dieux en haut et le régulateur du Feu ( $\mathrm{Li})$ qui rassemble les hommes, en bas, après que la communication qui reliait le ciel et la terre eut été

77. Cf. aussi sur ce thème, le Taishang laojun kaitian jing, 4a. 
coupée, est le contre-type des légendes des rois inventeurs, tout comme la barbarie est l'avers de l'Age d'Or ${ }^{78}$. Le mythe en effet s'interprète comme la transformation d'une chaine mythique traversant toute 1'Asie Orientale et Sud-pacifique. Dans ses diverses modulations, le mythe traite soit de la séparation du ciel et de la terre par la faute d'un monstre cannibale, provoquant l'apparition des arts du tissage chez les hommes, et des étoiles dans le ciel ${ }^{79}$, soit de l'apparition des singes, pendant sauvage de l'humain, après que le mil eut cessé de gonfler ${ }^{80}$, soit encore la séparation des hommes et des dieux à la suite de la fragmentation du riz ${ }^{81}$. En Chine même, le récit se retrouve sous diverses variantes : dans le my the de Chong et $\mathrm{Li}$, déjà cité, mais aussi dans la légende du Bouvier et de la Tisserande et enfin, sous une forme historicisée, dans les légendes des rois inventeurs qui séparent, grâce à un feu civilisateur, les hommes des animaux ${ }^{82}$. Ce n'est pas le lieu ici de reprendre ce volumineux dossier. Contentons-nous de constater que Age d'Or, sauvagerie, séparation du ciel et de la terre forment les pièces d'un puzzle. Cet âge sauvage, abîme de barbarie ou apothéose de l'Age d'Or que connut l'humanité primitive, est vue comme un chaos.

78. Il reste plusieurs versions anciennes du mythe : Shujing (Annales : 377-378); Guoyu (p. 203); Shanhä̈ing (16, p. 75b) ; Shïi , 25, p. 1257 ; Hanshu, 25, p. 1190. Pour une analyse du mythe, Cf. Maspero 1924, pp. 95-96, et Jean Lévi 1977. pp. 84-93. 14-15.

79. Cf. D.C. Graham 1954, p. 284 et les transformations du récit : pp. 192-194;

80. Cf. Ho Ting-jui 1972, pp. 275, 281-294, 329, 335, 338 et 352-353; Toichi Mobuchi 1964, p. 92. Pour une analyse partielle de ces récits, cf. Jean Lévi 1977, pp. 87-92.

81. Maspero 1924, pp. 95-96; 1971, pp. 259-161.

82. Il existe de très nombreuses versions et sous-versions du mythe du Bouvier et de la Tisserande. L'une des plus anciennes références au mythe est donnée par le Shiji (Cheu-king : 265). Cf. l'analyse de Kominami, p. 35. Pour des variantes de la légende, cf. Fan Ning 1955. pp. 21-33. 
Celui-ci évoque à son tour l'outre primordiale dont sont issus le ciel et la terre ${ }^{83}$. Mais celle-ci évoque aussi le riz originel, ou le mil gros comme une courge des temps où le ciel et la terre étaient encore proches l'un de l'autre, dans certains contes Tai et Atayal et que rappellent en un écho assourdi certaines versions du mythe du Bouvier et de la Tisserande 84 . De même, c'est sous forme d'une outre que se présente le produit incestueux d'une sœur et d'un frère, Nugua et Fuxi : masse de chair informe et sans orifices, dépourvue de membres et de tête, qu'il faudra découper en tranches pour que naisse l'humanité , comme le riz est fragmenté, comme la communication entre le ciel et la terre est tranchée pour qu'apparaissent le travail et la peine et avec eux la vieillesse, la mort et la décrépitude. Chaos, outre, œuf primordial, courge, boule de chair et grain miraculeux sont les modulations d'un même thème : celui de l'indistinction primitive.

On comprend que dans un tel système, revenir au chaos est synonyme de refuser les céréales, tout à la fois cause et produit de la séparation primordiale.

Ce modèle que les taoïstes contestent, plutôt que de l'attaquer de front, ils ont préféré, que ce soit consciemment ou non, jouer avec lui, le pervertir insidieusement. Mais attaquer ces vieux mythes étiologiques qui ont nourri toute la réflexion de l'orhtodoxie sur la place de l'homme dans l'univers, n'était-ce pas saper les fondements mêmes où elle puisait ses certitudes?

83. Il s'agit de la légende de Pangu. La version la plus célèbre est celle du Sanwu liji conservée au ch. 63 du Yuhan shanfang. Mais le thème est probablement ancien. On remarquera que le mythe cosmogonique a été approprié par les taoïstes. Cf. Maspero 1971 p. 374, et surtout la brillante analyse de K.M. Schipper (Schipper 1982, pp. 155-174).

84. La plus remarquable est celle de la collection Qingping shantang intitulée la "Rencontre de Dong Yong et de 1'Immortelle " (Dong Yong yu xian zhuan 董永遇仙傮) ( Qingping shantang hua ben, pp. 235-244). 


\section{BIBLIOGRAPHIE}

Sources chinoises

Annales de la Chine (trad. COUVREUR) Paris, Cathasia, 1950, (titre ori. Shujing)

Baihutong 白虎通, Shanghai Comm. Press, 1936.

Baopuzi 抱朴子, Shanghai, Comm. Press, 1936.

Bowuzhi 博物志, Taiwan, Comm. Press. 1960.

Cheu King (Trad. S. COUVREUR) Paris, Imprimerie de la Maison

Catholique, 1926 (titre orig. Shijing).

Concordance du Huang-t'ing king (établi par K.M. SCHIPPER),

Paris, EFEO, 1975.

Daozang 道藏, Shanghai, Hanfenlou, 1924 (réédit. photographique,

Taipei, Yiwen Yinshuguan, 1977).

Diwang shiji 帝王世纪, Shanghai, Comm. Press, 1936.

Dongxuan lingbao zhenren xiuxing yannian yicou fa

洞玄垔窝真人修行延年益軎法: DZ 1271 .

Fengsu tongyi 風唂通義 Shanghai, Comm. Press. 1936.

Guanzi 管 子, Taiwan, Comm. Press. 1956.

Guoyu 語, Shanghai, Comm. Press. 1946.

Gushi kao. 古史考 in TPYL

Gu xiaoshuo gouchen 古小説钩沈 in Luxun Quanii. VIII.

Hanfeizi 輅非子 Shanghai, Renmin chubanshi, 1974.

Hanshu 漠書, Pékin, zhonghua shuju, 1973.

Houhanshu 復漠害, Pékin, Zhonghua shuju, 1973. 
Huainanzi 淮南子, Shanghai, Comm. Press. 1937.

Huangting jing 黄庭 经 voir 《Concordance》.

Hunshu yisheng lu 混俗頭生镍 DZ 848 .

Laozi 老子 voir Pères

Liji 䄚記 voir 《Mémoires historiques》.

Lishi zhenxian tidao tongjian 歷世真仙體道通䍀: DZ 296.

Lie (le) Sien Tchouan Biographies d'immortels taoistes (trad.

KALTENMARK) Pékin, Publication du Centre Sinologique de

Paris, 1953 (titre ori. Liexian zhuan).

Liexian zhuan 列仙傅 (Cf. Lie Sien Tchouan).

Liezi 列子 : voir Pères.

Lushi 路史: SBBY (Shanghai, Zhonghua shuju).

Lüshi chunqiu 吕氏春秋, éd. SBCK (Shanghai, 1924).

Luxun quanji 鲁迅全集, Shanghai, renmin wenxue chubanshi, 1973.

Lunheng 論衡, Shanghai renmin chubanshi, 1973.

Mémoires historiques (trad. E. CHAVANNES) Paris, A. Maisonneuve, 1967 (Shiji)

Mémoires sur les bienséances et les cérémonies (trad. S.

COUVREUR) Paris, Cathasia, 1950.

Mengzi 孟子. Voir Oeuires.

Oeuvres (les) de Meng Tzeu (trad. S. COUVREUR), Paris, Cathasia

1950, (titre ori. Mengzi).

Pengzu shesheng yangxin lun. 彭祖㜔生养性論。DZ. 840 . Pères (les) du système taoïste (trad. L. Wieger), Paris, Cathasia, 1950.

Qingping shantang huaben 清平山堂話本. Shanghai.

Gudian wenxue chubanshi, Shanghai, 1957.

Sandong shuji zashuo 三洞樞機雜説. DZ 839

Sanguo zhi 三國志. Pékin, Zhonghua shuju, 1973.

Shanhaijing 山海经. éd. SBCK (Shanghai, Hanfanglou, 1924).

Shenxian zhuan 神仙傅, éd. Shuoku (Shanghai Wenmingshuju, 1914.) 
Shiji 史記 . Pékin. Zhonghua shuju, 1973.

Shijing 詩经. Voir Cheu King.

Shujing 書经. Voir Annales.

Shuyi jing 述異经. éd. Shuoku (Wenming shuju. Shanghai 1914).

Soushen $j i$ 搜神記. éd. Jindai bishu.

Taiping quangji 太平廣記. Pékin, Renmin wenxue chubanshi, 1956.

Taiping yulan 太平䘖筧. Pékin, Zhonghua shuju, 1960 (éd. photographique de l'éd. Hanfenlou).

Taiqingjing duangu fa 太清经断毂法. DZ 846.

Taishang dongyuan shenzhou jing 太上洞淵神咒经。 DZ. 335 .

Taishang Laojun kaitian jing 太上老君開天经.YJQQ 2 et $D Z .1437$.

Taishang Laojun yangsheng jue 太上老君养生孰. DZ 821 .

Taishang lingbao Shengxuan neijiaojing, zhonghe pin shuyishu 太上露赛异方内散经中和品述義端 $D Z$. 1122 .

Taishô yissai-kyo 大正新修大藏经

Tcheou (le) Li (trad. E. BIOT) Paris, Imprimerie Nationale, 1851.

Wenxian tong kao 文献通考 Shanghai. Comm. Press. 1936 (titre ori. Zhouli).

Wen xüan 文選 : Hongkong Comm. Press. 1974.

Wenzi zuanyi jijie 文子绩義集解，Shanghai，Guangyi shuju, 1936.

Taishang lingbao wufu xu 太上蛋覒五符序.DZ. 388. Wuyue chunqiu 吴越春秋. Shanghai, Comm. Press. 1937.

Xinyu 新語, ed. SBCK (Shanghai. . Hanfenlou, 1924).

Xuanjing yuanzhi fahui 玄经元指媵挥 in Daofa huiyuan 道法會元.DZ. 1220 .

Xunzi jianzhu 背子简注, Pékin, Zhonghua shuju 1973 .

Yangxing yanming $l u$ 养性延命淥, DZ. 838 .

Yiyuan 異苑 éd. Shuoku (Shanghai. Wenming shuju, 1914). 
Yunji qiqian 雲得七裁. DZ. 1032.

Zhenzhongji 枕中記. DZ. 837:

Zhuangzi 壮子. Voir Pères.

\section{Travaux et études}

B. BODDE

1975 Festivals in classical China, Princeton, Princeton University Press.

M. DETIENNE

1970 « La cuisine de Pythagore 》 Archives de Sociologie des Religions, 29, pp. 141-162.

DORE

1919 Recherches sur les superstitions en Chine, Shanghai variétés sinologiques, $\mathrm{n}^{\circ} 51$.

FAN NING

1955 《niulang zhi nü de yanbian》牛郎識女故 事的演变，Wenxue yichan zengkan, pp. 421-433.

J. GERNET

1960 "Les suicides par le feu chez les bouddhistes chinois $\mathrm{du} \mathrm{V}^{\mathrm{e}}$ au Xe siècle ». Mélanges IHEC. II. pp. 527-558.

D.C. GRAHAM

1954 Songs and stories of the ch'uan-Miao, Washington. Smithsonian,Institution.

M. GRANET

1953 Etudes sociologiques sur la Chine, Paris, PUF

1959 Danses et Légendes de la Chine ancienne, Paris, PUF de GROOT

1888 Fêtes annuellement célébrées à Emouï, Paris

1910 Religious system of the Chinese . Leiden HENSMAN S. M. KWOK-PING

1968 Hong-Kong tales spinner. Hong-Kong, Chinese Uni- 
HO TING-JUI

versity of Hong-Kong.

1972 Comparative study of myths and legends of Formosan Aborigenes, Taipei, « Asian and Social life monographs »18. Dongfang wenhua shuju.

M. KALTENMARK

1960 "Ling-bao. Note sur un terme du taoïsme religieux » Paris. Mélanges IHEC II, pp. 559-588.

\section{KOMINAMI}

1974 «Seibo to sichi densho » Tohogakuho 46. pp. 31-81.

\section{J. LEVI}

1977 «Le mythe de l'Age d'Or et les théories de l'évolution en Chine ancienne »L'homme XVII (1), pp. 73-103.

C. LEVI-STRAUSS

1964 Le cru et le cuit. Paris, Plon.

1967 Du miel aux Cendres, Paris, Plon.

H. MASPERO

1924 Les Contes mythologiques du Chou-king 》JA, 204, pp. 1-100.

1971 Essais sur le taoüsme et les religions chinoises, Paris, Gallimard.

\section{Des ROTOURS}

1963 «Quelques notes sur l'anthropologie en Chine » T'oung Pao L. pp. 386-427.

1968 «Encore quelques notes sur l'anthropologie en Chine» T'oung Pao LIV, pp. 1-49.

\section{K.M. SCHIPPER}

1965 L'empereur $W u$ des Han dans la légende taoïste. Paris, EFEO.

R. STEIN

1982 Le Corps taoïste . Paris, Fayard.

1970 «La légende du foyer dans le monde chinois » in

J. POUILLON \& P. MANDARE, eds. Echanges et 
communications. Mélanges offerts à Claude LéviStrauss à l'occasion de son 60 anniversaire. Paris, Mouton.

1971 Les fêtes de cuisine du taoïsme religieux : Paris, Annuaire du Collège de France, pp. 431-440.

1972 «Spéculations mystiques et thèmes relatifs aux cuisines du taoïsme » Paris, Annuaire du Collège de France, pp. 489-499.

\section{TOICHI MABUCHI}

1964 «Tales concerning the origin of grains in the insular areas of Eastern and south-eastern Asia » Asian Folklore Studies, XXIII (1). pp. 1-92.

\section{WENWU}

1975 "Mawangdui sanhao hanmu beihua daoyintu de chubu yaniiu》馬王堆三号漠墓帛畫尊引圆的初步研究 Mawangdui beishu "que gu shi qi pian》kao.6 馬王堆 帛害《卻谷食氧篇》考

\section{TABLE DES ABREVIATIONS}

Annales : Annales de la Chine

Comm. Press : Shangwu yinshu guan

Concordance : Concordance du Huang-t'ing king

$D Z$ : Daozang

$J A:$ Journal Asiatique

Mémoires : Mémoires sur les biensésances et les cérémonies

Oeuvres :Oeuvres (les)de Meng-Tzeu

Pères : Pères (les) du système taoiste

SBBY : Sibubeiyao 
SBCK : Sibu congkan

Shengxuan jing : Taishang lingbao neijiao jing zhonghe pin shuyishu TPGJ : Taiping guangji

TPYL : Taiping yulan

$Y J Q Q:$ Yunji qiqian

Jean LEVI

C.NRS 